\title{
Synaptic Mechanisms of Direction Selectivity in Primary Auditory Cortex
}

\author{
Chang-quan Ye, ${ }^{1}$ Mu-ming Poo, ${ }^{1,2}$ Yang Dan, ${ }^{2,3}$ and Xiao-hui Zhang ${ }^{1}$ \\ ${ }^{1}$ Institute of Neuroscience and State Key Laboratory of Neuroscience, Shanghai Institutes for Biological Sciences, Chinese Academy of Sciences, Shanghai \\ 200031, China, ${ }^{2}$ Division of Neurobiology, Department of Molecular and Cell Biology and Helen Wills Neuroscience Institute, University of California, \\ Berkeley, California 94720, and ${ }^{3}$ Howard Hughes Medical Institute, University of California, Berkeley, California 94720
}

Frequency modulation (FM) is a prominent feature in animal vocalization and human speech. Although many neurons in the auditory cortex are known to be selective for FM direction, the synaptic mechanisms underlying this selectivity are not well understood. Previous studies of both visual and auditory neurons have suggested two general mechanisms for direction selectivity: (1) differential delays of excitatory inputs across the spatial/spectral receptive field and (2) spatial/spectral offset between excitatory and inhibitory inputs. In this study, we have examined the contributions of both mechanisms to FM direction selectivity in rat primary auditory cortex. The excitatory and inhibitory synaptic inputs to each cortical neuron were measured by in vivo whole-cell recording. The spectrotemporal receptive field of each type of inputs was mapped with random tone pips and compared with direction selectivity of the neuron measured with FM stimuli. We found that both the differential delay of the excitatory input and the spectral offset between excitation and inhibition are positively correlated with direction selectivity of the neuron. Thus, both synaptic mechanisms are likely to contribute to FM direction selectivity in the auditory cortex. Finally, direction selectivity measured from the spiking output is significantly stronger than that based on the subthreshold membrane potentials, indicating that the selectivity is further sharpened by the spike generation mechanism.

\section{Introduction}

Frequency-modulated auditory stimuli sweeping across wide spectral ranges are prevalent in the natural environment. The direction of frequency modulation (FM) carries important information in animal communication (Winter et al., 1966; Kanwal et al., 1994; Wang, 2000) and human speech (Lindblom and Studdert-Kennedy, 1967; Gold and Morgan, 2000; Zeng et al., 2005). Neurons in several stages of the auditory pathway, including inferior colliculus (Nelson et al., 1966; Gordon and O'Neill, 1998; Fuzessery et al., 2006), auditory thalamus (O'Neill and Brimijoin, 2002), and the primary auditory cortex (A1) (Suga, 1965b; Mendelson and Cynader, 1985; Zhang et al., 2003), are known to exhibit FM direction selectivity, but the underlying circuit mechanisms remain unclear.

Previous studies have suggested two general mechanisms well suited for shaping direction selectivity. First, the latency of excitatory inputs changes systematically with the stimulus location (visual) or frequency (auditory) (Fig. 1 $A, B$, differential latency model). The moving visual stimulus or FM sound sweep in the preferred direction activates long-latency inputs before shortlatency inputs, evoking higher responses via temporal summa-

Received June 30, 2009; revised Dec. 14, 2009; accepted Dec. 17, 2009.

This work was supported by Knowledge Innovation Program of the Chinese Academy of Sciences Grant KSCX2YW-R-29 and Major State Basic Research Program of China Grant 2006CB806600. Y.D. and M.-m.P. were supported in part by grants from the United States National Institutes of Health.

Correspondence should be addressed to either of the following: Yang Dan, Department of Molecular and Cell Biology, University of California, Berkeley, CA 94720-3200, E-mail:ydan@berkeley.edu; or Xiao-hui Zhang, Institute of Neuroscience, Chinese Academy of Sciences, Shanghai 200031, China, E-mail: xhzhang@ion.ac.cn.

DOI:10.1523/JNEUROSCI.3088-09.2010

Copyright $\odot 2010$ the authors $\quad 0270-6474 / 10 / 301861-08 \$ 15.00 / 0$ tion. In contrast, stimulus in the opposite direction activates temporally dispersed inputs, evoking lower responses (Fig. 1C). Although this mechanism has been demonstrated in the primary visual cortex (V1) (Livingstone, 1998; Priebe and Ferster, 2005; Priebe and Ferster, 2008), whether it operates in the auditory cortex is unclear. Another "asymmetric inhibition" mechanism involves inhibitory inputs preferentially localized to one side of the excitatory region (Fig. $1 D, E$ ). Whereas the preferred stimulus sweeps across the excitatory region before entering the inhibitory sideband, thus evoking large responses, stimulus in the opposite direction activates the inhibitory input first, effectively suppressing excitatory responses (Fig. $1 F$ ). Although asymmetric suppressive sidebands have been demonstrated in both V1 (Livingstone, 1998; Murthy and Humphrey, 1999) and A1 (Suga, 1965b; Shamma et al., 1993; Nelken and Versnel, 2000; Razak and Fuzessery, 2006), the synaptic nature of the suppression is only beginning to be characterized (Zhang et al., 2003; Wu et al., 2008).

In this study, we used in vivo whole-cell recording in rat A1 to examine the role of both differential latency and asymmetric inhibition in FM direction selectivity. The excitatory and inhibitory inputs to each neuron were separated by recording the responses at several holding voltages, and their spectrotemporal receptive fields (STRFs) were mapped with random tone pips (deCharms et al., 1998; Theunissen et al., 2000). We found that, for excitatory inputs, the latency shifts systematically with stimulus frequency in a manner consistent with direction selectivity of the neuron, whereas inhibitory inputs show less consistent latency shift. Furthermore, we found a significant difference between the spectral tuning of excitatory and inhibitory inputs, and the spectral offset 
A

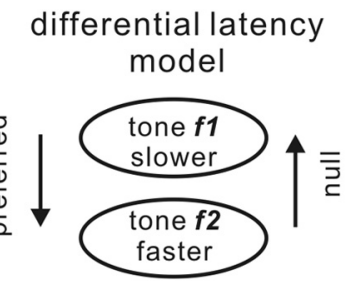

B

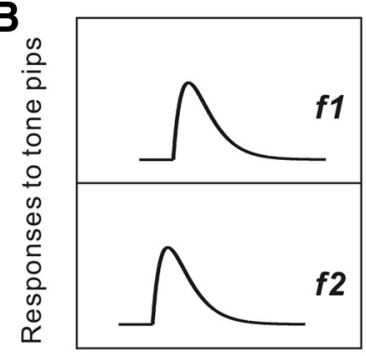

C

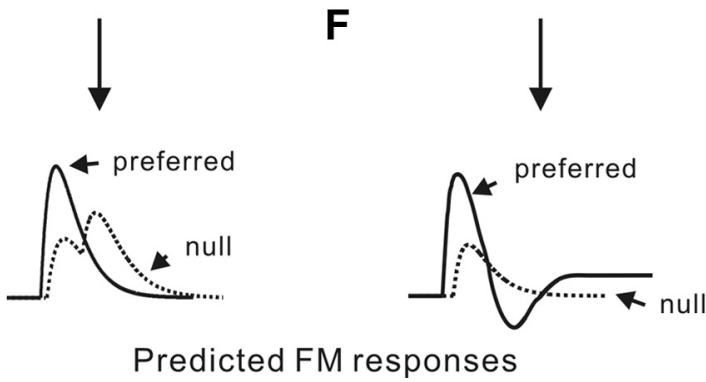

Figure 1. Schematicillustration of two potential circuit mechanisms for direction selectivity. $A-C$, Differential latency mechanism. Tone pips at frequencies $f 1$ and $f 2$ evoke responses with long and short latencies, respectively $(\boldsymbol{B})$. When FM sound sweeps from $f 1$ to $f 2$ (preferred direction), the responses to $f 1$ and $f 2$ arrive simultaneously, giving rise to a large summed response ( $\boldsymbol{C}$, solid line). For null direction, the responses to $f 1$ and $f 2$ arrive at different times, resulting in a summed response with low amplitude ( $\boldsymbol{C}$, dotted line). $\boldsymbol{D}-\boldsymbol{F}$, Asymmetric inhibition mechanism. Tone $f 1$ evokes an excitatory response, and tone $f 2$ evokes a delayed inhibitory response $(\boldsymbol{E})$. For preferred direction, FM sound sweeps across the excitatory region first and then evokes a delayed inhibition, so the inhibitory response fails to suppress the excitatory response ( $\boldsymbol{F}$, solid line). For null direction, inhibitory response is evoked first, which effectively suppresses the subsequent excitatory response ( $\boldsymbol{F}$, dotted line).

is correlated with the FM direction selectivity of the cell. Thus, both differential latency and asymmetric inhibition mechanisms may contribute to FM direction selectivity in A1. Finally, direction selectivity measured from the spike rate is significantly stronger than that measured from the subthreshold responses, consistent with findings in V1 (DeAngelis et al., 1993; Emerson, 1997; Baker, 2001; Conway and Livingstone, 2003; Priebe and Ferster, 2005).

\section{Materials and Methods}

\section{Animal preparation}

Adult male Sprague Dawley rats (7-10 weeks of age, 200-350 g) were used in this study. Animals were anesthetized with pentobarbital (initially $50 \mathrm{mg} / \mathrm{kg}$, i.p., maintained at $6 \mathrm{mg} / \mathrm{h}$ ). After the animal was mounted in a stereotaxic device, a craniotomy was performed and dura matter was removed to allow access to the A1 in the left hemisphere. CSF was released at the medulla level. Throughout the experiment, the core body temperature $\left(37.5-38.5^{\circ} \mathrm{C}\right.$, maintained by a custom-made electric blanket), heart rate, and EEG were continuously monitored to assess the level of anesthesia. The animal use protocol was approved by the Animal Research Advisory Committee of Shanghai Institutes for Biological Sciences, Chinese Academy of Sciences.

\section{Whole-cell recording}

For whole-cell recordings, the glass microelectrode was filled with the

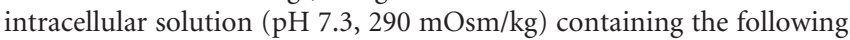

(in mM): $136 \mathrm{~K}$-gluconate, $4 \mathrm{KCl}, 10 \mathrm{HEPES}, 20 \mathrm{Na}$-gluconate, $2 \mathrm{MgSO}_{4}$, and 0.2 EGTA. For the experiments measuring the excitatory and inhibitory synaptic inputs, QX-314 [N-(2,6-dimethylphenylcarbamoylmethyl)triethylammonium bromide] ( $5 \mathrm{~mm}$ ) was added in the intracellular solution to block $\mathrm{Na}^{+}$channels. The recording pipette was advanced at a speed of $5-10 \mu \mathrm{m} / \mathrm{s}$ at $45^{\circ}$ from the horizontal. Most of the cells were recorded 150-1000 $\mu \mathrm{m}$ from the pial surface. The whole-cell recording was made with Axopatch 200A (Molecular Devices). Signals were filtered at 2 $\mathrm{kHz}$ (low pass) and sampled at $10 \mathrm{kHz}$. To measure the excitatory and inhibitory synaptic inputs, the postsynaptic current responses to each stimulus (tone pips or FM sweeps) were recorded at two to three holding potentials, which were corrected for a calculated liquid junction potential (Barry, 1994) of $16 \mathrm{mV}$. A total of 57 cells were included in this study, with 11 cells recorded without QX-314 (to allow measurement of spiking responses, as shown in Figs. 2, 7) and 46 recorded with QX-314 (to isolate the synaptic conductances). Although we have measured the excitatory and inhibitory STRFs for these 46 cells, only STRFs exhibiting clear tuning were used for additional analyses (see below in Data analysis, Cell selection). As a result, the excitatory STRFs of 38 cells were used for the analysis in Figure 4; the inhibitory STRFs of 37 cells were used in Figure 5. The 29 cells with well tuned excitatory and inhibitory STRFs (including those in both Figs. 4, 5) were used in Figure 6.

\section{Auditory stimulation}

Sound stimuli were generated and delivered by an electrostatic speaker ED1 through a hollow ear bar to the right ear using TDT system 3 (Tucker Davis Technologies). The frequencies of the tone pips varied from 512 to $48 \mathrm{kHz}$ with $0.1,0.2$, or 0.3 octave interval. Each tone pip lasted for $25 \mathrm{~ms}$ with $5 \mathrm{~ms}$ linear ramp. The FM stimuli swept between 0.5 and $48 \mathrm{kHz}$ at a speed of 30-90 octaves/s in either upward or downward direction, delivered in a pseudorandom order at $2-4 \mathrm{~Hz}$. Direction selectivity of the recorded neuron was determined at several intensities of the FM stimuli.

\section{Data analysis}

Measurement of direction selectivity. Direction selectivity index (DI) was define as $\left(P_{\text {up }}-P_{\text {down }}\right) /\left(P_{\text {up }}+P_{\text {down }}\right)$, where $P_{\text {up }}$ and $P_{\text {down }}$ are the spike rates, the amplitudes of subthreshold membrane potentials (measured under current clamp), or amplitudes of postsynaptic currents (measured under voltage clamp with holding potential of -70 $\mathrm{mV}$ ) in response to the upward and downward FM sounds, respectively. In experiments without blocking action potentials, we separated the spikes by a high-pass filter (Carandini and Ferster, 2000) offline. The peristimulus time histogram (PSTH) was plotted from 60 trials with $5 \mathrm{~ms}$ bins. With the subtraction of the baseline firing rate, spike rate within $120 \mathrm{~ms}$ from the onset of upward or downward sweep was counted as $P_{\text {up }}$ or $P_{\text {down }}$. For measuring the amplitude of subthreshold responses, the membrane potentials after removal of spikes were averaged (Carandini and Ferster, 2000), and the peak amplitude of depolarization was measured.

Measurements of excitatory and inhibitory conductances. The separation of excitatory and inhibitory conductances followed the method described by Wehr and Zador (2003). Briefly, the evoked synaptic current $I_{\text {syn }}$ was computed as follows:

$$
I_{\text {syn }}(t)=\frac{R_{s}+R_{m}}{R_{m}} \Delta I(t)
$$

where $\Delta I(t)$ is the current recorded during sound stimulus minus the baseline current measured within $50 \mathrm{~ms}$ before stimulus onset, and $R_{s}$ and $R_{m}$ are the series and input resistances, respectively. The membrane potential $V_{m}(t)$ is determined by

$$
V_{m}(t)=V_{\text {hold }}-I(t) R_{s}
$$

Based on Ohm's law, $I_{\text {syn }}(t)=G_{\text {syn }}(t)\left(V_{m}(t)-E_{\text {syn }}(t)\right)$, the total synaptic conductance $G_{\text {syn }}(t)$ and total synaptic reversal potential $E_{\text {syn }}(t)$ were 
A

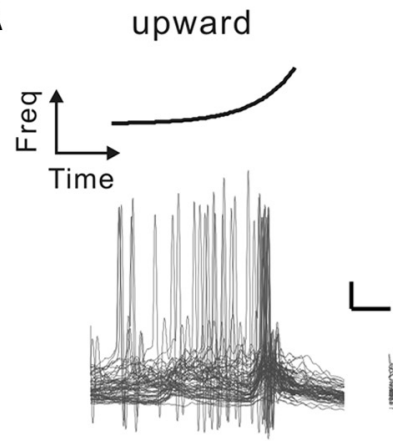

B

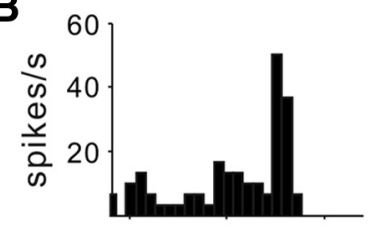

C

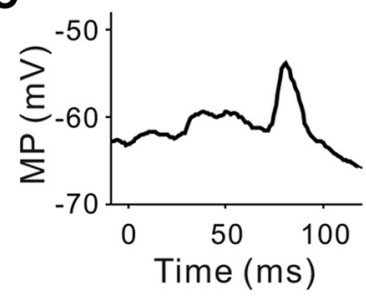

downward
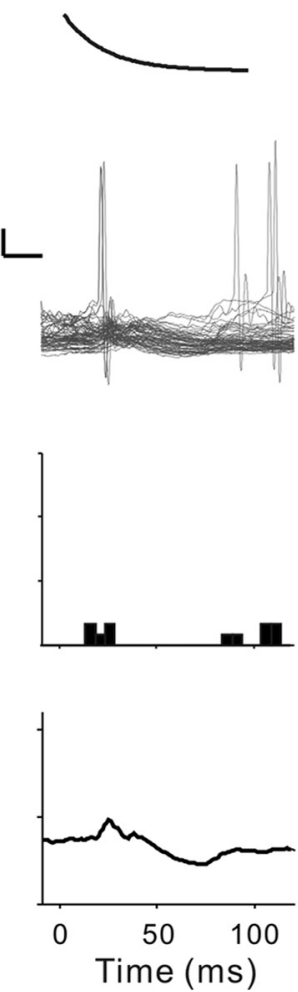

D

upward downward
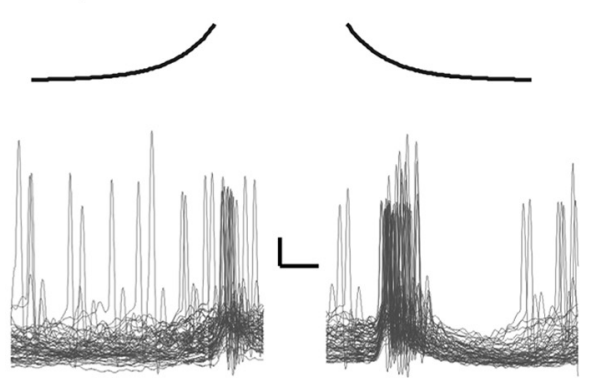

E
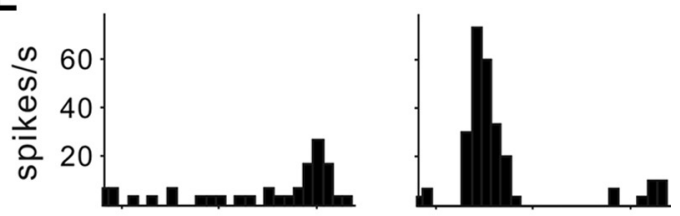

$\mathbf{F}$

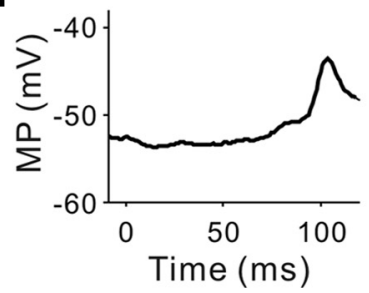

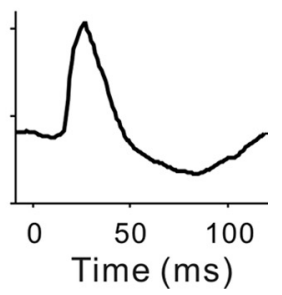

Figure 2. Whole-cell recording from direction-selective neurons in A1.A-C, An example neuron that preferred upward FM stimulus. D-F, Another neuron that preferred downward FM stimulus. $A, D$, Superimposed traces depict membrane potential changes evoked by 60 repeats of upward and downward FM stimuli (at 70 octaves/s). Each trace represents response in one trial. Calibration: $10 \mathrm{mV}, 20 \mathrm{~ms} . \boldsymbol{B}, \boldsymbol{E}$, PSTHs of spikes extracted from the raw traces. $\boldsymbol{C}, \boldsymbol{F}$, Subthreshold membrane potentials extracted from the raw traces, averaged across all trials. The Dls of these two cells are 1 and -0.62 (based on spike rate) or 0.54 and -0.18 (based on amplitude of subthreshold responses), respectively.

measured as the slope and intercept, respectively, of the linear regression of $I_{\text {syn }}$ versus $V_{\mathrm{m}}$. The excitatory and inhibitory conductances, $G_{e}(t)$ and $G_{i}(t)$, were then computed as follows:

$$
\begin{gathered}
G_{i}(t)=G_{\text {syn }}(t) \frac{E_{e}-E_{\text {syn }}(t)}{E_{e}-E_{i}}, \\
G_{e}(t)=G_{\text {syn }}(t)-G_{i}(t) .
\end{gathered}
$$

Here $E_{e}$ and $E_{i}$ are the reversal potentials for excitatory and inhibitory inputs, respectively. For our internal solution, $E_{e}=0 \mathrm{mV}$ and $E_{i}=-85$ $\mathrm{mV}$ (Wehr and Zador, 2003). We found that, in our experiments, the linear regression (see Fig. $3 B$, solid line) was very close to the mean $I_{\text {syn }}$ at each $V_{\mathrm{m}}$ (gray circles). This indicates that the conductance is primarily voltage independent, consistent with the basic assumption used in this analysis.

Cell selection. Only cells exhibiting well tuned excitatory or inhibitory STRFs were included in the analysis. For each type of inputs, we summed its STRF over the period of $10-70 \mathrm{~ms}$ after the stimulus onset to obtain its spectral tuning. This tuning was then fitted by a Gaussian function. The STRF was included in additional analyses only if the Gaussian fitting reached $R^{2}>0.2$.

Prediction of DI based on Fourier transform of STRFs. We performed Fourier transformation of excitatory or inhibitory STRFs over the period of $10-70 \mathrm{~ms}$ after stimulus onset (see Figs. $4 B, 5 B$ ), and DI was predicted as

$$
\mathrm{DI}=\frac{\left(w_{1}+w_{3}\right)-\left(w_{2}+w_{4}\right)}{\sum w_{i}},
$$

where $w_{1}, w_{2}, w_{3}$, or $w_{4}$ are Fourier amplitudes in each of the four quadrants (see Figs. $4 B, 5 B$ ), averaged over the range of 16.7-40 cycles/s for the temporal axis and $0.3-1.5$ cycles/octave for the spectral axis.
Prediction of FM response from STRF. The excitatory or inhibitory conductance change evoked by an FM sweep was predicted by convolution of the STRF and the FM stimulus at the given sweep rate. This is equivalent to shifting the response at each frequency by a time delay determined by the sweep rate before summing the responses across frequencies (supplemental Fig. 3, available at www.jneurosci.org as supplemental material). The onset latency of evoked conductance was defined by the time point when the evoked change was 1.96 times of SD of the baseline. To predict the FM sweep-evoked responses measured under current or voltage clamp, we used the following equations:

$$
\begin{gathered}
V_{m}=\frac{G_{e} E_{e}+G_{i} E_{i}+G_{\text {rest }} E_{\text {rest }}}{G_{e}+G_{i}+G_{\text {rest }}}, \\
I(t)=G_{\text {syn }}(t) \frac{V_{\text {hold }}-E_{\text {syn }}(t)}{\left(R_{m}+R_{s}\right) / R_{m}+G_{\text {syn }}(t) R_{s}} .
\end{gathered}
$$

\section{Results}

Neuronal direction selectivity in A1 of adult rats was examined by using whole-cell recording method (see Materials and Methods). In a previous study using whole-cell recordings, the strongest FM direction selectivity of rat $\mathrm{A} 1$ neurons was found at a sweep rate of 70 octaves/s when excitatory currents were measured (Zhang et al., 2003). The result of our pilot experiment measuring direction selectivity as a function of sweep rate (supplemental Fig. 1, available at www.jneurosci.org as supplemental material) was consistent with this finding. To measure FM direction selectivity, we thus presented FM sweeps at a speed of 70 octaves/s in both upward and downward directions (duration of $94 \mathrm{~ms} / \mathrm{sweep}$ ), and membrane potential responses were recorded under current 
clamp (Fig. $2 A, D$ ). We found that some neurons preferred upward (Fig. 2A-C) and others preferred downward (Fig. 2D-F) FM stimuli. DI based on the spiking response is defined as $\left(P_{\text {up }}-\right.$ $\left.P_{\text {down }}\right) /\left(P_{\text {up }}+P_{\text {down }}\right)$, where $P$ represents the spike rate within $120 \mathrm{~ms}$ from the sound onset after subtracting the baseline firing rate (Fig. $2 B, E$ ). After removing the spikes (see Materials and Methods), we measured direction selectivity of the subthreshold membrane potential responses based on the peak amplitude of depolarization. The DI of subthreshold responses showed the same sign as spiking responses (Fig. 2C,F). For this population of neurons in which both spiking and subthreshold responses were measured $(n=11)$, the DIs based on the spike rate and the subthreshold response were found to be highly correlated [correlation coefficient $(c c)=0.59, p=0.0004$ ] (see Fig. 7).

\section{Measurements of synaptic conductance}

To examine whether the synaptic mechanisms illustrated in Figure 1 contribute to FM direction selectivity of A1 neurons, we next measured the excitatory and inhibitory conductances in each neuron under voltage clamp. To reduce the contamination from voltage-dependent $\mathrm{Na}^{+}$channel conductance, we added QX-314 in the internal solution. Because QX-314 effectively blocked action potentials, DI was computed only from the subthreshold responses in these experiments. Supplemental Figure 2 (available at www.jneurosci.org as supplemental material) shows the DI distribution of 46 cells recorded with QX-314 addition, in which 25 cells exhibited DI $>0.1$ and 11 cells exhibited DI $<-0.1$.

To measure the excitatory and inhibitory conductances evoked by each stimulus (either tone pip or FM sweep), we repeated each stimulus for multiple times and recorded the synaptic currents under voltage clamp at two or three different holding potentials. Figure $3 A$ shows the responses of an example cell evoked by a tone pip recorded at three holding potentials. The recorded current at each time point was plotted against the holding potential, and the linear fit was made for all the data points (Fig. 3B). The excitatory and inhibitory conductances (Fig. 3C) can be computed from the slope and the intercept of the linear fit (Borg-Graham et al., 1998; Wehr and Zador, 2003; Zhang et al., 2003) (see Materials and Methods). In the following analyses, we focused on the stimulus-evoked change in each conductance, defined as the measured conductance minus the baseline conductance of a $50 \mathrm{~ms}$ period before stimulus onset.

\section{Differential latency of synaptic input}

To obtain the STRF of each synaptic input, we measured the excitatory and inhibitory conductances evoked by random tone pip stimuli and computed the STRF using reverse correlation (deCharms et al., 1998; Theunissen et al., 2000). Figure $4 A$ shows the excitatory STRF of an example neuron. The latency of excitatory responses decreased systematically with the stimulus frequency (Fig. 4A, circles: peak responses at each frequency), suggesting that this neuron preferred upward FM sweeps (Fig. $1 A-C)$. With linear convolution of the excitatory STRF and the FM stimuli, the predicted excitatory conductance changes evoked by FM sweeps appeared indeed larger for the upward sweep than the downward sweep (Fig. 4C). This could be attributed to different temporal alignments of the responses at different frequencies (supplemental Fig. 3, available at www. jneurosci.org as supplemental material). The recorded excitatory conductance change of this neuron evoked by the FM stimuli confirmed this prediction (Fig. 4D). The FM direction selectivity of this cell measured under current clamp (DI $=0.24)$ was also consistent with the prediction based on the excitatory STRF. For the
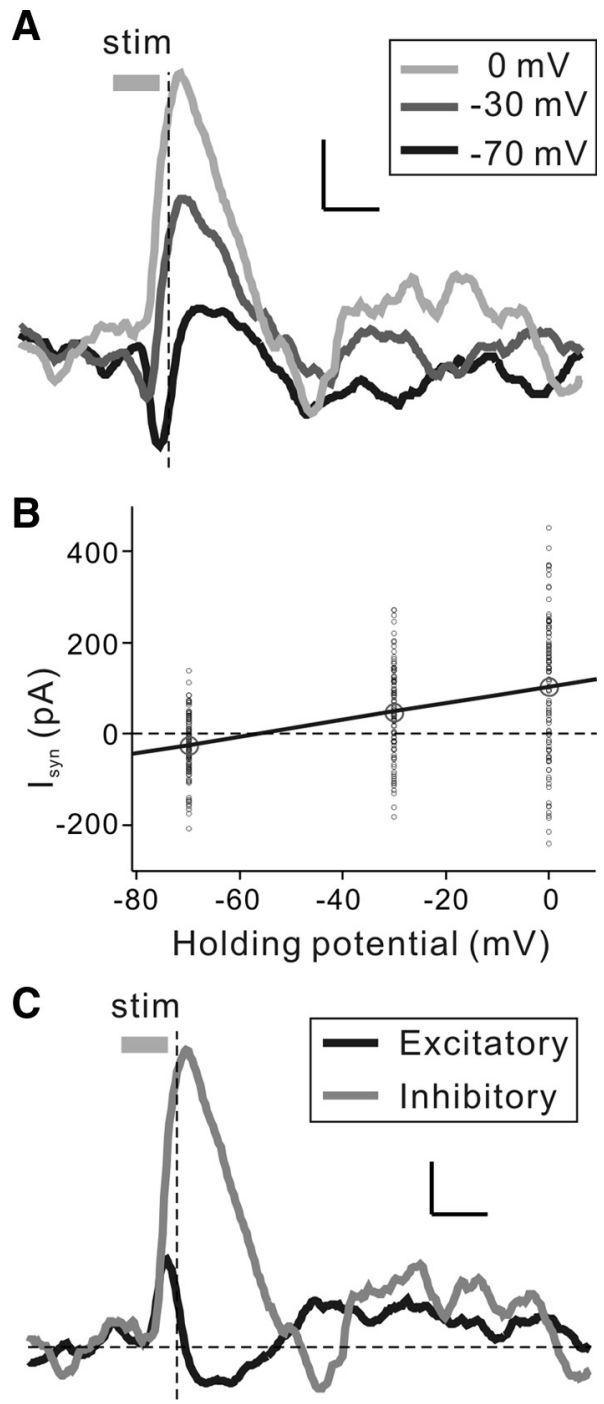

Figure 3. Separation of excitatory and inhibitory conductances under voltage clamp. $\boldsymbol{A}$, Synaptic currents evoked by a tone pip (duration indicated by horizontal gray bar), recorded at three holding potentials. Each trace was averaged from 100 trials. Calibration: 30 pA, 30 ms. B, Synaptic current amplitudes at $30 \mathrm{~ms}$ after stimulus onset (indicated by vertical dashed line in A) plotted against the clamping membrane potentials. Each point represents data from one trial. Solid line, linear regression; bigger gray circles, averaged value across trials. The slope and $x$-intercept of the linear regression represent the total conductance (1.83 nS) and synaptic reversal potential $(-56.6 \mathrm{mV})$, respectively. C, Separated excitatory (black) and inhibitory (gray) conductances in response to the tone pip. Calibration: $0.3 \mathrm{nS}, 30 \mathrm{~ms}$.

population of neurons tested $(n=10)$, the correlation coefficient between the direction selectivity of the predicted excitatory conductance and that of the measured conductance (at the sweep rate of 70 octaves/s) was 0.37 .

To quantify direction selectivity of the excitatory input attributable to the spectrotemporal profile of its STRF, we also performed Fourier transform of the STRF. As shown by the example neuron in Figure $4 A$, an STRF with shorter latency at higher frequencies exhibits higher amplitude in the first (top right) and third (bottom left) quadrants than that in the second and fourth quadrants of the Fourier spectrum (Fig. $4 B$ ). This asymmetry of the Fourier spectrum has been used extensively to predict neuronal direction selectivity (Adelson and Bergen, 1985; DeAngelis et al., 1993; Priebe and Ferster, 2005) (see Materials and Methods). The population result of this analysis is shown in Figure $4 E$. The predicted DI of the excitatory input was highly correlated with 
A $08717 \mathrm{c} 2$, excitatory

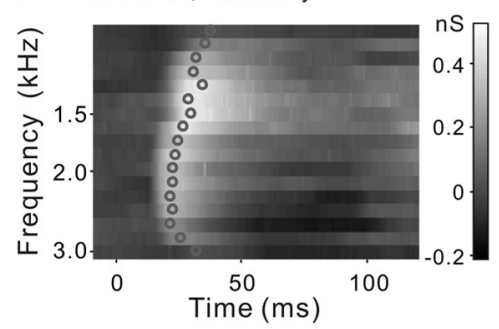

B

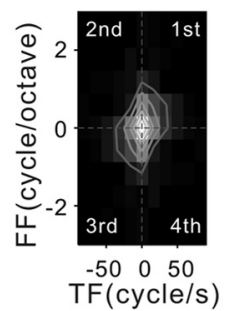

D Actual

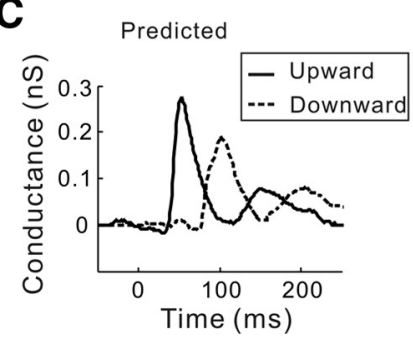

E
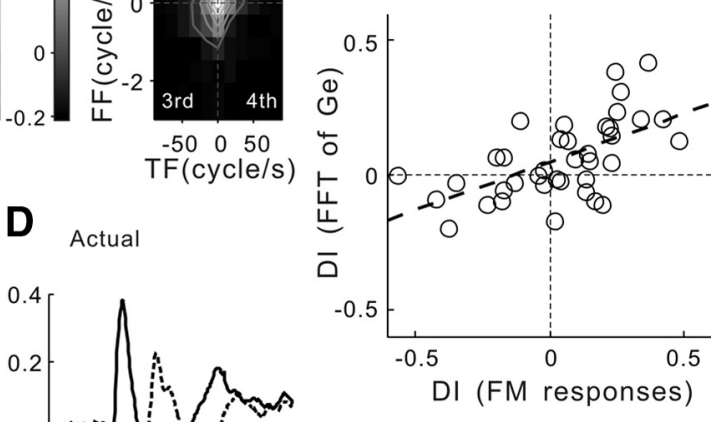

sweeps (Fig. 5C). This prediction was confirmed by the measured inhibitory conductance changes evoked by FM sweeps (Fig. 5D). The Fourier transform of the inhibitory STRF was also primarily symmetric (Fig. $5 B$ ), predicting low direction selectivity of the inhibitory input. For the population of cells, the predicted DI of the inhibitory input based on Fourier transform was less correlated with the measured DI of the neurons ( $\mathrm{cc}=0.39$, $p=0.02, n=37)($ Fig. 5E).

\section{Spectral offset between excitatory and inhibitory inputs}

We next tested whether asymmetric inhibition (Fig. 1D-F) contributes to direction selectivity. To obtain spectral tuning of excitatory and inhibitory inputs, we integrated each STRF over the period 10-70 $\mathrm{ms}$ after stimulus onset (Fig. 6A, between dashed lines). For the example neuron shown in Figure $6 B$, the inhibitory input was tuned to higher frequencies than the excitatory input. As shown in the linear prediction of the conductance changes evoked by FM stimuli (Fig. $6 C, D$, left columns), the onset of excitation preceded onset of inhibition for upward but not for downward sweeps (see Materials and Methods). This direction-dependent difference in onset latency was confirmed by the measured conductance changes evoked by FM sweeps (Fig. 6C,D, right columns). Such relative onset latency between excitation and inhibition predicts that this cell prefers the upward FM sweep. The DI (0.48) of this cell measured under current clamp was indeed consistent with this prediction. For the population of cells tested $(n=10)$, we measured the difference of the relative excitation/inhibition onset latency between the upward and downward sweeps. We found that such upward-downward difference from the predicted FM sound responses agreed well with that from measured responses $(\mathrm{cc}=0.58)$, suggesting that the spectral offset between the excitatory and inhibitory tuning curves contributes to onset latency difference between the excitation and inhibition in FM responses.

To quantify the difference in spectral tuning between the excitatory and inhibitory inputs, we defined the spectral offset the DI of the neuron measured with FM stimuli ( $c c=0.59, p=$ $0.0001, n=38$ ). Together, these results suggest that differential latency of the excitatory input (Fig. $1 A-C$ ) contributes significantly to the FM direction selectivity of A1 neurons.

In contrast to the excitatory STRF shown in Figure $4 A$, the inhibitory STRF of the same cell did not show a consistent latency shift (Fig. 5A). As a result, the linear prediction of the inhibitory conductance changes was similar for upward and downward

as the distance in the center of mass between the inhibitory and excitatory tuning curves (Fig. $6 B$, arrowheads). For the 29 cells with well tuned excitatory and inhibitory inputs, we found a significant correlation between the spectral offset and the DI of the neuron measured with FM sweeps ( $c c=0.43, p=0.02)$. This result indicates that the spectral offset between excitation and inhibition also contributes to the FM direction selectivity of A1 neurons. 

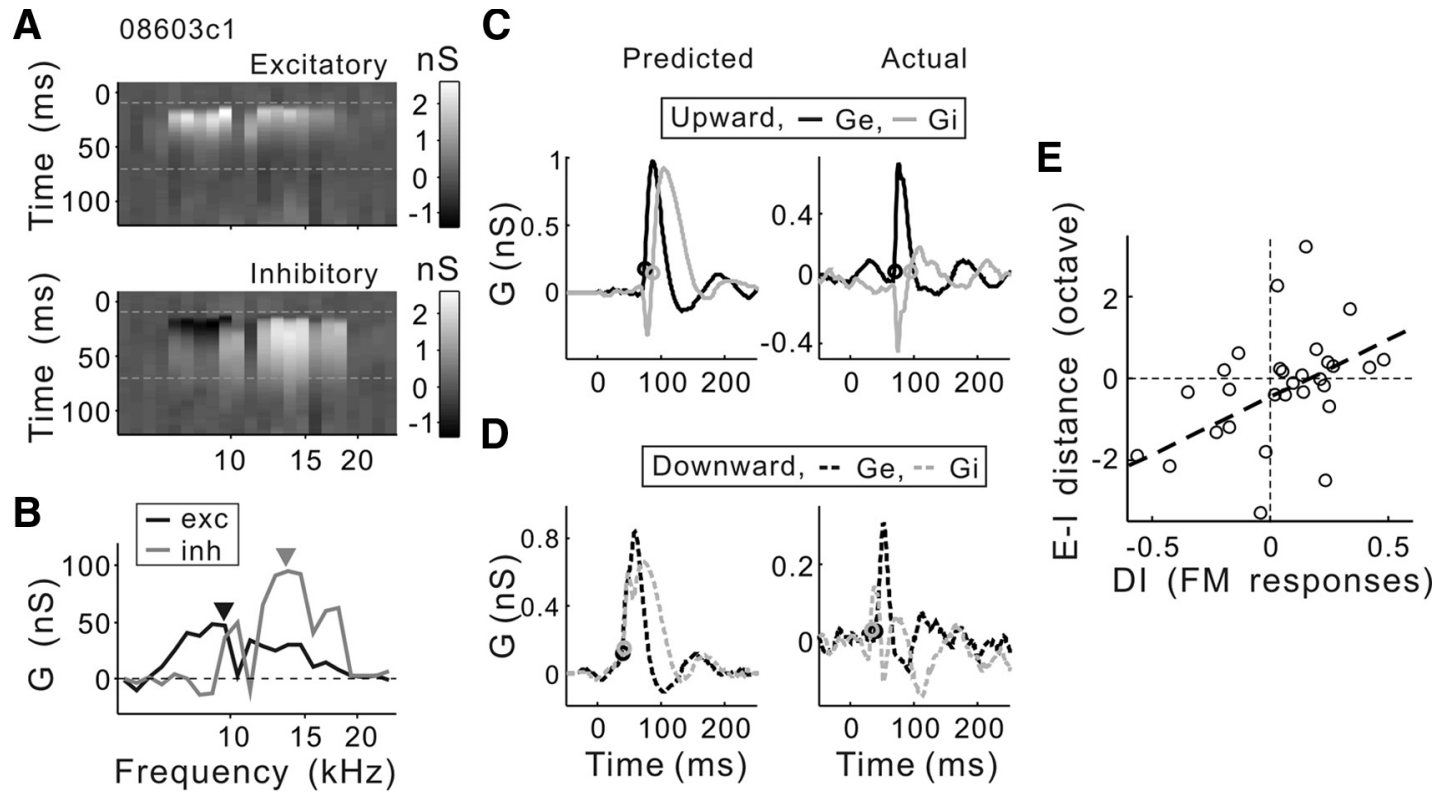

Figure 6. Spectral offset between excitatory and inhibitory STRFs. $\boldsymbol{A}$, STRFs of excitatory and inhibitory inputs of an example neuron. $\boldsymbol{B}$, Integrated excitatory (black) or inhibitory (gray) conductances over $10-70 \mathrm{~ms}$ after stimulus onset (between dashed lines in $A$ ), as a function of the sound frequency. Arrowheads indicate the center of mass of each function. The distance between inhibitory and excitatory tuning curves (E-I distance) is measured by the spectral distance between the two arrowheads, a value of 0.40 octave for this cell. C, D, Predicted (left) and actual (right) excitatory $\left(G_{e}\right)$ and inhibitory $\left(G_{i}\right)$ conductances evoked by upward $(\boldsymbol{C})$ and downward $(\boldsymbol{D})$ FM sweeps, respectively. Circles denote the onset time of excitatory or inhibitory conductance. For upward sweep, $G_{e}$ precedes $G_{i}$ in the prediction and actual measurement by 12 and $24 \mathrm{~ms}$, respectively. For downward FM sweep, the values were 2 and $-7 \mathrm{~ms}$, respectively. $E$, E-I distance versus DI of the neuron measured by FM sweeps. Each circle represents one cell $(n=29)$. Thick dashed line represents linear fit of the data $(c c=0.43, p=0.02)$.

Note that the contribution of both the differential latency and the spectral offset to direction selectivity depends on the sweep rate. For the two cells shown in Figures 4-6, we predicted the excitatory and inhibitory conductance changes evoked by FM stimuli at a range of sweep rates based on the STRF of each input. As shown in supplemental Figure 4 (available at www.jneurosci. org as supplemental material), the difference between the predicted responses to upward and downward FM sweeps diminished at very high sweep rates. To test how well the linear model incorporating both excitatory and inhibitory mechanisms can account for FM direction selectivity at various sweep rates, we used the predicted conductance changes to compute the synaptic currents measured at a holding potential of $-70 \mathrm{mV}$, evoked by FM sounds at sweep rates ranging between 30 and 90 octaves/s (see Materials and Methods) (supplemental Fig. 5, available at www.jneurosci.org as supplemental material). The DI based on the predicted responses was then compared with the measured DI of a population of cells in which a range of sweep rates were tested experimentally. We found that the predicted DI agreed well with measured DI at 50 octaves/s $(\mathrm{cc}=0.56, p=0.02)$ and 70 octaves $/ \mathrm{s}(\mathrm{cc}=0.52, p=0.03)$, less well at 30 octaves $/ \mathrm{s}(\mathrm{cc}=0.41$, $p=0.1)$, and very poorly at 90 octaves/s $(c c=0.002, p=1)$. Thus, although the direction selectivity at 50-70 octaves/s are well accounted for by the linear model, the mechanisms may be much more nonlinear at much faster sweep rates.

\section{Sharpening of direction selectivity by spike generation}

Previous studies have shown that the spike threshold serves to sharpen the feature selectivity of visual cortical neurons, including orientation tuning and direction selectivity (DeAngelis et al., 1993; Emerson, 1997; Baker, 2001; Conway and Livingstone, 2003; Priebe and Ferster, 2005). We thus compared the FM direction selectivity of A1 neurons measured from the subthreshold membrane potential and from the spike rate (see Materials and

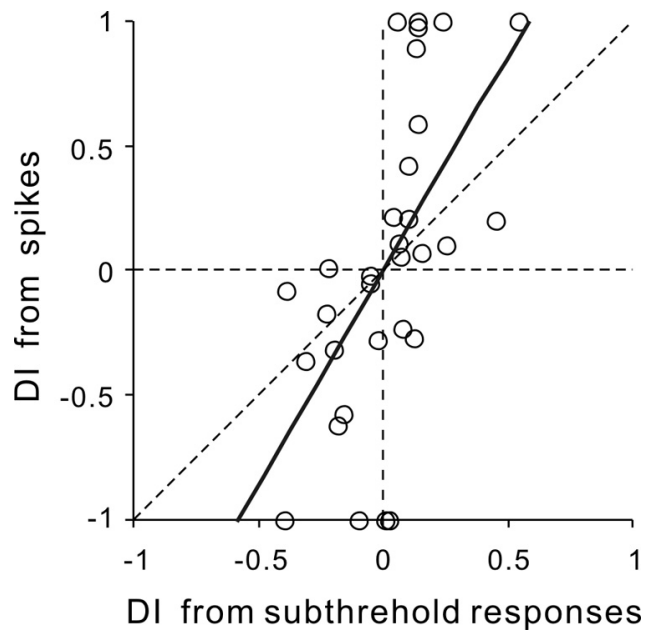

Figure 7. Sharpening of direction selectivity by spike generation. DI measured from spike rate is plotted against that from subthreshold membrane potentials. Data are from 11 neurons, each measured at multiple sound intensities. Each circle represents a test at a particular intensity $(n=31)$. Solid line indicates linear fit (slope of $1.72, y$-intercept $=-0.0002$, $c=0.59$, $p=0.0004)$.

Methods). As shown in Figure 7, the two measurements of DI are highly correlated, but the DI measured from spike rates was significantly stronger than that measured from subthreshold responses $(\mathrm{cc}=0.59, p=0.0004)$. Thus, the spike generation mechanism further sharpens direction selectivity in the auditory cortex.

\section{Discussion}

In this study, we have measured the excitatory and inhibitory conductances to each A1 neuron and mapped their STRFs. We found that both the differential latency of excitatory input and 
the spectral offset between excitation and inhibition are correlated with direction selectivity of A1 neuron in response to FM sweeps. Thus, our results have provided direct evidence that the two circuit mechanisms that have been proposed for direction selectivity in general contribute to direction selectivity in A1 for FM sounds at a considerable range of sweep rates. Because direction selectivity is already present in earlier stages of the auditory pathway, including auditory thalamus (O'Neill and Brimijoin, 2002), inferior colliculus (Nelson et al., 1966; Gordon and O'Neill, 1998; Fuzessery et al., 2006), and possibly at even lower auditory nuclei (Gittelman et al., 2009), the two synaptic mechanisms in A1 together with the spike generation mechanism are likely to enhance direction selectivity of cortical neurons.

Extracellular recordings in cat and monkey A1 (Phillips et al., 1985; deCharms et al., 1998; Atencio et al., 2007) and in bat inferior colliculus (Andoni et al., 2007) showed that direction selectivity of neurons can be predicted by their STRFs. A recent study in bat auditory cortex suggested that direction selectivity may arise from facilitatory interactions between a pair of tones at particular spectral and temporal intervals (Razak and Fuzessery, 2008). These findings all point to the possibility that differential latencies of inputs tuned to different frequencies may contribute to direction selectivity, although the nature of synaptic inputs could not be ascertained from these studies using extracellular recordings. In the present work, separation of excitatory and inhibitory inputs revealed that the differential delay of the excitatory input is indeed well correlated with the FM direction selectivity (Fig. 4). Although the inhibitory STRF shows a similar tendency, the correlation with the neuronal direction selectivity is considerably weaker (Fig. 5). Thus, differential latency of the excitatory input seems to play a more important role in FM direction selectivity of A1 neurons. Spectrotemporally inseparable receptive fields have also been observed in the inferior colliculus (Langner et al., 1987; Andoni et al., 2007), and intracellular recordings showed that the responses to FM sweeps can be primarily accounted for by temporal summation of postsynaptic potentials with different latencies (Voytenko and Galazyuk, 2007). Thus, the differential latency mechanism may operate in multiple stages of the auditory pathway to shape the neuronal FM direction selectivity. However, additional studies are necessary to determine whether the differential latency in A1 originates from precortical or intracortical connections. We also note that, although the effect of differential latency on direction selectivity can be well illustrated by a simple linear model (Fig. 4) (supplemental Fig. 4, available at www.jneurosci.org as supplemental material), this does not exclude the contribution of nonlinear mechanisms to FM direction selectivity.

Many previous studies have suggested the role of asymmetric inhibitory sidebands in FM direction selectivity (Suga, 1965a,b; Fuzessery and Hall, 1996; Gordon and O'Neill, 1998; Zhang et al., 2003; Razak and Fuzessery, 2006). Although stimuli in these sidebands are known to suppress the spiking response evoked by an optimal stimulus, results from the extracellular recording experiments could not distinguish whether the suppression is mediated by the increase in inhibition or the reduction of excitation (Wehr and Zador, 2005). A recent study showed that local blockade of $\mathrm{GABA}_{\mathrm{A}}$ receptors in $\mathrm{A} 1$ reduced or eliminated direction selectivity in $\sim 50 \%$ cells (Razak and Fuzessery, 2009), indicating that direction selectivity of some cortical neurons is significantly shaped by intracortical inhibition. Moreover, using whole-cell recording, Wu et al. (2008) showed that the frequency tuning of inhibitory input was broader than that of excitatory input, which could serve to sharpen the frequency tuning of cortical neurons.
The present study further indicates that, for some Al neurons, the inhibitory inputs are asymmetric and such asymmetric sidebands of the inhibition contribute to FM direction selectivity, consistent with the finding of Zhang et al. (2003). A notable caveat is that our experiments were performed under pentobarbital anesthesia, which is known to affect GABAergic synaptic transmission. In future studies it would be important to determine the contribution of asymmetric inhibition to direction selectivity in awake animals. Asymmetric inhibition has also been suggested in direction-selective neurons in the visual cortex (Livingstone, 1998). In the somatosensory cortex, direction selectivity was shown to emerge from a direction-dependent temporal shift between excitatory and inhibitory inputs (Wilent and Contreras, 2005), although it is unclear whether the latter is attributable to a spatial offset between excitation and inhibition.

In summary, we have characterized two features of the excitatory and inhibitory receptive fields of A1 neurons, which contribute to FM direction selectivity. These mechanisms, together with the spike generation threshold (Fig. 7), appear to shape direction selectivity in various sensory modalities along multiple stages of the neuronal pathways.

\section{References}

Adelson EH, Bergen JR (1985) Spatiotemporal energy models for the perception of motion. J Opt Soc Am A 2:284-299.

Andoni S, Li N, Pollak GD (2007) Spectrotemporal receptive fields in the inferior colliculus revealing selectivity for spectral motion in conspecific vocalizations. J Neurosci 27:4882-4893.

Atencio CA, Blake DT, Strata F, Cheung SW, Merzenich MM, Schreiner CE (2007) Frequency-modulation encoding in the primary auditory cortex of the awake owl monkey. J Neurophysiol 98:2182-2195.

Baker CL Jr (2001) Linear filtering and nonlinear interactions in directionselective visual cortex neurons: a noise correlation analysis. Vis Neurosci 18:465-485.

Barry PH (1994) JPCalc, a software package for calculating liquid junction potential corrections in patch-clamp, intracellular, epithelial and bilayer measurements and for correcting junction potential measurements. J Neurosci Methods 51:107-116.

Borg-Graham LJ, Monier C, Frégnac Y (1998) Visual input evokes transient and strong shunting inhibition in visual cortical neurons. Nature 393:369-373.

Carandini M, Ferster D (2000) Membrane potential and firing rate in cat primary visual cortex. J Neurosci 20:470-484

Conway BR, Livingstone MS (2003) Space-time maps and two-bar interactions of different classes of direction-selective cells in macaque V-1. J Neurophysiol 89:2726-2742.

DeAngelis GC, Ohzawa I, Freeman RD (1993) Spatiotemporal organization of simple-cell receptive fields in the cat's striate cortex. II. Linearity of temporal and spatial summation. J Neurophysiol 69:1118-1135.

deCharms RC, Blake DT, Merzenich MM (1998) Optimizing sound features for cortical neurons. Science 280:1439-1443.

Emerson RC (1997) Quadrature subunits in directionally selective simple cells: spatiotemporal interactions. Vis Neurosci 14:357-371.

Fuzessery ZM, Hall JC (1996) Role of GABA in shaping frequency tuning and creating FM sweep selectivity in the inferior colliculus. J Neurophysiol 76:1059-1073.

Fuzessery ZM, Richardson MD, Coburn MS (2006) Neural mechanisms underlying selectivity for the rate and direction of frequency-modulated sweeps in the inferior colliculus of the pallid bat. J Neurophysiol 96:1320-1336.

Gittelman JX, Li N, Pollak GD (2009) Mechanisms underlying directional selectivity for frequency-modulated sweeps in the inferior colliculus revealed by in vivo whole-cell recordings. J Neurosci 29:13030-13041.

Gold B, Morgan N (2000) Speech and audio signal processing: processing and perception of speech and music. New York: Wiley.

Gordon M, O’Neill WE (1998) Temporal processing across frequency channels by FM selective auditory neurons can account for FM rate selectivity. Hear Res 122:97-108. 
Kanwal JS, Matsumura S, Ohlemiller K, Suga N (1994) Analysis of acoustic elements and syntax in communication sounds emitted by mustached bats. J Acoust Soc Am 96:1229-1254.

Langner G, Schreiner C, Merzenich MM (1987) Covariation of latency and temporal resolution in the inferior colliculus of the cat. Hear Res 31:197-201.

Lindblom BE, Studdert-Kennedy M (1967) On the role of formant transitions in vowel recognition. J Acoust Soc Am 42:830-843.

Livingstone MS (1998) Mechanisms of direction selectivity in macaque V1. Neuron 20:509-526.

Mendelson JR, Cynader MS (1985) Sensitivity of cat primary auditory cortex (AI) neurons to the direction and rate of frequency modulation. Brain Res 327:331-335.

Murthy A, Humphrey AL (1999) Inhibitory contributions to spatiotemporal receptive-field structure and direction selectivity in simple cells of cat area 17. J Neurophysiol 81:1212-1224.

Nelken I, Versnel H (2000) Responses to linear and logarithmic frequencymodulated sweeps in ferret primary auditory cortex. Eur J Neurosci 12:549-562.

Nelson PG, Erulkar SD, Bryan JS (1966) Responses of units of the inferior colliculus to time-varying acoustic stimuli. J Neurophysiol 29:834-860.

O’Neill WE, Brimijoin WO (2002) Directional selectivity for FM sweeps in the suprageniculate nucleus of the mustached bat medial geniculate body. J Neurophysiol 88:172-187.

Phillips DP, Mendelson JR, Cynader MS, Douglas RM (1985) Responses of single neurones in cat auditory cortex to time-varying stimuli: frequencymodulated tones of narrow excursion. Exp Brain Res 58:443-454.

Priebe NJ, Ferster D (2005) Direction selectivity of excitation and inhibition in simple cells of the cat primary visual cortex. Neuron 45:133-145.

Priebe NJ, Ferster D (2008) Inhibition, spike threshold, and stimulus selectivity in primary visual cortex. Neuron 57:482-497.

Razak KA, Fuzessery ZM (2006) Neural mechanisms underlying selectivity for the rate and direction of frequency-modulated sweeps in the auditory cortex of the pallid bat. J Neurophysiol 96:1303-1319.

Razak KA, Fuzessery ZM (2008) Facilitatory mechanisms underlying selectivity for the direction and rate of frequency modulated sweeps in the auditory cortex. J Neurosci 28:9806-9816.
Razak KA, Fuzessery ZM (2009) GABA shapes selectivity for the rate and direction of frequency-modulated sweeps in the auditory cortex. J Neurophysiol 102:1366-1378.

Shamma SA, Fleshman JW, Wiser PR, Versnel H (1993) Organization of response areas in ferret primary auditory cortex. J Neurophysiol 69:367-383.

Suga N (1965a) Responses of cortical auditory neurones to frequency modulated sounds in echo-locating bats. Nature 206:890-891.

Suga N (1965b) Functional properties of auditory neurones in the cortex of echo-locating bats. J Physiol 181:671-700.

Theunissen FE, Sen K, Doupe AJ (2000) Spectral-temporal receptive fields of nonlinear auditory neurons obtained using natural sounds. J Neurosci 20:2315-2331.

Voytenko SV, Galazyuk AV (2007) Intracellular recording reveals temporal integration in inferior colliculus neurons of awake bats. J Neurophysiol 97:1368-1378.

Wang X (2000) On cortical coding of vocal communication sounds in primates. Proc Natl Acad Sci U S A 97:11843-11849.

Wehr M, Zador AM (2003) Balanced inhibition underlies tuning and sharpens spike timing in auditory cortex. Nature 426:442-446.

Wehr M, Zador AM (2005) Synaptic mechanisms of forward suppression in rat auditory cortex. Neuron 47:437-445.

Wilent WB, Contreras D (2005) Dynamics of excitation and inhibition underlying stimulus selectivity in rat somatosensory cortex. Nat Neurosci 8:1364-1370

Winter P, Ploog D, Latta J (1966) Vocal repertoire of the squirrel monkey (Saimiri sciureus), its analysis and significance. Exp Brain Res 1:359-384.

Wu GK, Arbuckle R, Liu BH, Tao HW, Zhang LI (2008) Lateral sharpening of cortical frequency tuning by approximately balanced inhibition. Neuron 58:132-143.

Zeng FG, Nie K, Stickney GS, Kong YY, Vongphoe M, Bhargave A, Wei C, Cao $\mathrm{K}$ (2005) Speech recognition with amplitude and frequency modulations. Proc Natl Acad Sci U S A 102:2293-2298.

Zhang LI, Tan AY, Schreiner CE, Merzenich MM (2003) Topography and synaptic shaping of direction selectivity in primary auditory cortex. Nature 424:201-205. 